\title{
Validity assessment of the symptom checklist SCL-90-R and shortened versions for the general population in Ukraine
}

\author{
Yuliia Sereda ${ }^{{ }^{*}}$ and Serhii Dembitskyi ${ }^{2}$
}

\begin{abstract}
Background: The Symptom Checklist-90-Revised (SCL-90-R) is a widely used symptomatic distress questionnaire. A translated version of the SCL-90-R has been applied in Ukrainian general population surveys several times but has not yet been validated in this country. The SCL-90-R and its short versions (BSI-53, SCL-27, BSI-18, SCL-14 and SCL-9-K) were investigated in order to comparatively assess their properties and applications in Ukraine.

Methods: Secondary analysis of three nationally representative cross-sectional surveys (1997, 1999 and 2014) using SCL-90-R was applied. Two thousand sixty nine respondents participated in 2014; the sample size for the 1997 and 1999 surveys was 1810 respondents per wave. Statistical data analysis is based on calculating internal consistencies with Cronbach's Alpha, confirmatory factor analysis, nonparametric correlations and effect sizes for the equivalence of the full and short versions.
\end{abstract}

Results: The scales of SCL-90-R and its shortened versions showed equally high internal consistencies. With regard to factorial validity, 2014 data confirmed the dimensional structure of all versions. Unsatisfactory results were found in 1997 and 1999 for SCL-90-R and in 1997 for SCL-27, based on the Chi-square criterion (X2/degrees of freedom > 5), though other indexes suggested satisfactory model fit (RMSEA $<0.06 ; \mathrm{CFI}, \mathrm{TLI}>0.95$ ). Analysis of the equivalence of shortened and full versions of the SCL-90-R has shown the presence of small effect sizes.

Conclusion: BSI-18 and SCL-9-K are recommended for use in general population surveys as more economical versions of SCL-90-R. Both versions revealed satisfactory validity in 1997, 1999 and 2014.

Keywords: SCL-90-R, Short versions, Mental disorders, Symptomatic distress, Self-report questionnaire

\section{Background}

Symptom Checklist-90-Revised (SCL-90-R) is a widely used questionnaire developed by Leonard R. Derogatis [1] to determine a number of psychological symptoms. In Ukraine, SCL-90-R was first used in the study "Mental health of children after the Chernobyl disaster" [2]. Later, it was applied in three surveys with samples that were representative of the entire population (1997, 1999 and 2014), but it has not yet been validated.

SCL-90-R includes 90 symptoms and evaluates nine symptomatic dimensions: somatization, obsessive-compulsive disorder, interpersonal sensitivity, depression, anxiety,

\footnotetext{
* Correspondence: yulia.v.sereda@gmail.com

${ }^{1}$ Senior Scientific Associate, Department for Monitoring of Social and Economic Transformations, Institute for Economics and Forecasting, National Academy of Sciences, Kiev, Ukraine

Full list of author information is available at the end of the article
}

hostility, phobic anxiety, paranoid ideation, and psychoticism [1]. Given the demand for briefer measures to be used as a screening tool for psychiatric disorders, shortened versions of SCL-90-R were developed, such as BSI-53 [3], SCL-27 [4], BSI-18 [5], SCL-14 [6, 7] and SCL9-K $[6,8]$. BSI-53 includes all nine symptomatic dimensions with a reduced number of symptoms, whereas SCL-27, BSI-18 and SCL-14 have both reduced factor structures and reduced numbers of items. SCL-9-K is the shortest measure, including nine symptoms within a single dimession (general severity factor). Numbers of indicators for symptomatic dimensions in SCL-90-R and its shortened versions are presented in Table 1.

The vast majority of psychometric studies studies on SCL-90-R were conducted on clinical samples, such as patients of mental health centers and agencies $[9,10]$, 
Table 1 Dimensional structure and items of the SCL-90-R and its shortened versions

\begin{tabular}{|c|c|c|c|c|c|c|c|c|c|c|c|}
\hline \multicolumn{2}{|c|}{ SCL-90-R } & \multicolumn{2}{|l|}{ BSI-53 } & \multicolumn{2}{|c|}{$\mathrm{SCL}-27$} & \multicolumn{2}{|l|}{ BSI-18 } & \multicolumn{2}{|c|}{ SCL-14 } & \multicolumn{2}{|c|}{ SCL-K-9 } \\
\hline Scale & Indicators & Scale & Indicators & Scale & Indicators & Scale & Indicators & Scale & Indicators & Scale & Indicators \\
\hline SOMA & $\begin{array}{l}1,4,12,27,40,42,48,49,52,53 \\
56,58\end{array}$ & SOMA & $4,12,40,48,49,52,56$ & VEG & $4,39,40,48,49,53$ & SOMA & $12,40,48,52,56,58$ & VEG & $42,52,56,58$ & & \\
\hline OCD & $3,9,10,28,38,45,46,51,55,65$ & OCD & $9,28,45,46,51,55$ & DYS & $9,14,51,55$ & & & & & & \\
\hline INT & $6,21,34,36,37,41,61,69,73$ & INT & $34,37,41,69$ & SOP & $37,41,61,69$ & & & & & & \\
\hline DEPR & $\begin{array}{l}5,14,15,20,22,26,29,30,31,32 \\
54,71,79\end{array}$ & DEPR & $15,29,30,32,54,79$ & DEP & $15,30,54,59$ & DEPR & $15,29,30,32,54,79$ & DEP & $26,28,30,54,77,79$ & & \\
\hline ANX & $2,17,23,33,39,57,72,78,80,86$ & ANX & $2,23,33,57,72,78$ & & & ANX & $2,33,57,72,78,86$ & & & & \\
\hline HOST & $11,24,63,67,74,81$ & HOST & $11,24,63,67,74$ & & & & & & & & \\
\hline $\mathrm{PHOB}$ & $13,25,47,50,70,75,82$ & $\mathrm{PHOB}$ & $13,47,50,70,75$ & AGO & $13,25,33,50,82$ & & & AGO & $13,25,47,82$ & & \\
\hline PARA & $8,18,43,68,76,83$ & PARA & $8,18,43,76,83$ & MIS & $18,68,76,83$ & & & & & & \\
\hline PSYC & $7,16,35,62,77,84,85,87,88,90$ & PSYC & $7,77,85,88,90$ & & & & & & & & \\
\hline ADD & $19,44,59,60,64,66,89$ & ADD & $19,44,59,89$ & & & & & & & & \\
\hline GSI & all above & GSI & all above & GSI & all above & GSI & all above & GSI & all above & GSI & $\begin{array}{l}24,28,31,34,43,57 \\
58,75,77\end{array}$ \\
\hline
\end{tabular}

SOMA Somatization, OCD Obsessive-Compulsive Disorder, INT Interpersonal Sensitivity, DEPR Depression, ANX Anxiety, HOST Hostility, PHOB Phobic Anxiety, PARA Paranoid Ideation, PSYC Psychoticism, GSI Global Severity Index, DEP Depressive Symptoms, DYS Dysthymic Symptoms, VEG Vegetative Symptoms, AGO Agoraphobic Symptoms, SOP Symptoms of Social Phobia, MIS Symptoms of Mistrust 
patients with depression [11], patients undergoing personality-centered therapy [12], forcibly hospitalized patients with mental disorders [13], adults and adolescents hospitalized with crisis intervention [14], substance abusers [15], patients with panic disorders [16], veterans undergoing psychiatric treatment [17], patients waiting for bariatric surgery [18], volunteers for drug trials [19] etc. A number of studies estimated properties of SCL-90-R on non-clinical samples, in particular those representative of the entire population or of certain communities; such studies were conducted in Canada [20], Denmark [21], Finland [22], Germany [23], Hungary [24], Japan [25], Italy [26], Norway [27], Thailand [28] and the USA [29].

Overall, there is increasing agreement on the multidimensional nature of the SCL-90-R, although various solutions from bifactor structure [24] to the nine original dimesions $[16,22,23,25]$ have been reported. A few studies support the unidimensional structure of the SCL-90-R as broad construct of distress [21, 28]. Weakness of the validity of SCL-90-R is explained by different reasons, including limitations of sample design and statistical measures. A German study revealed that subscale internal reliabilities are better for clinical samples when compared to nonclinical samples, which might result in revision of the SCL90- $\mathrm{R}$ for the general population [23]. R. Urbán et al. [24] highlighted that the vast majority of studies inappropriately used methods considering responses on a linear scale instead of an ordinal scale, and implemented the maximum likelihood estimator for measuring factor validity, which underestimates the fit of the models in confirmatory factor analysis, resulting in weak structural validity.

Comparative validation of the SCL-90-R and its shortened versions requires further investigation. While the vast majority of papers focus on the full version of the SCL-90-R, Müller et al. [30] examined the validity of eleven shortened versions and recommended SCL-10S as an instrument to measure psychological distress. Recently, Prinz et al. compared the psychometric properties of five shortened versions and concluded that BSI-18 appears to be the most economical variant and most clinically meaningful instrument [6]. None of the comparative validation studies of the SCL-90-R were conducted on non-clinical samples.

Given that previous studies did not come up with a single solution regarding factor validity of the SCL-90-R, we attempt to investigate SCL-90-R in order to comparatively assess its properties and application in Ukraine. Moreover, we concentrate on the comparative validation of SCL-90-R and its five shortened versions (BSI-53, SCL-27, BSI-18, SCL-14, SCL-9-K) in order to assess the extent to which they can reliably measure psychological distress as well as certain distress subscales. In particular, we examine which shortened version provides superior reliability, validity and practical utility in national monitoring surveys with representative samples. Our choice of shortened versions is driven by the evidence that BSI-53 and SCL-27 showed superior discriminant validity while BSI-18, SCL-14 and SCL-9-K demonstrated better performance regarding the general severity factor among the shortest versions in the previous studies $[6,30]$.

\section{Methods \\ Design}

The research is based on a secondary analysis of data collected by the Institute of Social Sciences, National Academy of Sciences of Ukraine (a social monitoring "Ukrainian Society" for 1997 and 1999, principal investigator Prof. Dr. Evgeniy Golovakha), as well as the joint monitoring of the Ukrainian Institute for Social Research after A. Yaremenko, Social Monitoring Center and the Department for Monitoring of Social and Economic Transformations, Institute for Economics and Forecasting, National Academy of Sciences of Ukraine (2014 study, principal investigator Olga Balakireva). In 1997 and 1999, 1810 respondents were interviewed; 2069 respondents were interviewed in 2014. Each of the three crosssectional studies is representative of the main sociodemographic characteristics of the adult population of Ukraine. In the 1997 and 1999 arrays the sex ratio was $45 \%$ male and $55 \%$ female, and the mean age was 45 years; in the 2014 array, $44 \%$ were male and $56 \%$ were female, and the mean age was 46 years. The 2014 study included 24 regions of Ukraine and Kiev, while in 1997 and 1999, 24 regions of Ukraine, Kiev and the Crimea were included. In all three studies data collection was administered through a face-to-face questionnaire. SCL-90-R was first translated and adapted for Ukraine by Dr. Nataliia Panina for a survey of mothers evacuated from Pripyat, Chernobyl in 1986 [2]. The adequacy of the Ukrainian and Russian translation to the English version was assessed through a back-translation by a professional translator.

\section{Tools}

In all three studies, the questionnaire SCL-90-R was completed as one section of a general questionnaire that included a wide range of social, political and economic aspects. The questionnaire was translated into both Ukrainian and Russian, as different languages were used for different regions of the country. Shortened versions of the symptomatic questionnaire (BSI-53, SCL-27, BSI-18, SCL-14, SCL-9-K) were calculated on the basis of SCL90-R questions during the secondary analysis.

\section{Statistical analysis}

Included reliability assessment of SCL-90-R subscales, factorial validity of symptomatic measurements and equivalence of individual variants of SCL-90-R. All methods were applied for all three studies (1997, 1999 and 2014). 
To assess the reliability of individual symptomatic measures and the Global Severity Index (GSI) in all six versions (SCL-90-R, BSI-53, SCL-27, BSI-18, SCL-14, SCL-9-K) Cronbach's alpha coefficients were calculated. Values of the coefficient that were higher than 0.7 were considered acceptable [31].

To confirm the factor validity of symptomatic measurements of the full and abbreviated versions of SCL-90-R, confirmatory factor analysis (CFA) was carried out. Given that all indicators have ordinal scales, a Diagonally Weighted Least Squares method (DWLS) was used to estimate the parameters of the CFA, which allows estimation of robust standard errors and correction of the test statistics. Missing values (up to $5 \%$ ) were excluded. To assess the quality of the factor models the following indices have been estimated: $\chi^{2}$ (Minimum Function Chi-square), RMSEA (The Root Mean Square Error of Approximation), CFI (Comparative fit index) and TLI (Tucker-Lewis index). An acceptable model fit was considered $\chi 2$ /degrees of freedom < 5; RMSEA < 0.06; and CFI, TLI > 0.95 [32].

Since the distribution of all indicators of symptomatic measurements and GSI in the full and shortened versions of the SCL-90-R deviated from normal, nonparametric methods were used for the analysis of equivalence. To analyze the equivalence of the full and shortened versions of SCL-90-R, median and interquartile distances were estimated, the statistical significance of the median differences was calculated on the basis of the Wilcoxon median test, and effect sizes and Spearman's Rho correlations were defined. We used Vargha and Delaney's A effect sizes, according to which a small effect is over 0.56; a medium effect is over 0.64 , and a large effect is over 0.71 [33]. The size of the correlations was based on the following interpretation limits: rho $<0.30$, small correlation; $0.30>$ rho $<0.50$, medium correlation and rho $>0.50$, large correlation [34].

The equivalence of different versions of the SCL-90-R was also evaluated in the context of the size difference of the group with a high risk of psychological distress in the general population, depending on the method, or in other words, the extent to which the prevalence of "probable cases" differs. According to Derogatis' criterion for the general population, if the GSI has a $T$-value $\geq 63$, such individuals may be characterized by the presence of severe symptoms of distress [35]. It is also common to use the criterion of GSI > 1 to determine the proportion of people with severe symptoms of distress [36].

$\mathrm{R}$ (package «lavaan» for CFA) and SPSS, version 20 were used for the statistical analysis.

\section{Results}

Reliability

In the 1997 study Cronbach's alpha coefficients for different symptomatic measurements ranged from 0.59 (depressive symptoms in SCL-27) to 0.96 (GSI in the SCL-90-R); in 1999 - from 0.63 (depressive symptoms in SCL-27) to 0.97 (GSI in the SCL-90-R), and in 2014 - from 0.66 (depressive symptoms in SCL-27) to 0.98 (GSI in SCL- 90-R) (see Table 2). Cronbach's alpha coefficients of below an acceptable level of reliability were observed for interpersonal sensitivity in BSI-53 and symptoms of social phobia in the SCL-27 (1997 and 1999), for hostility and phobic anxiety in the 1997 study (in BSI-53), for psychoticism in 1997 and 1999 (in BSI-53), for symptoms of mistrust in 1997 and 1999 (in SCL-27) and for agoraphobic symptoms in 1997

Table 2 Reliability of the SCL-90-R subscales and the shortened versions BSI, SCL-27, BSI-18, SCL-14 and SCL-K-9 in the Ukrainian general population

\begin{tabular}{|c|c|c|c|c|c|c|c|c|c|c|c|c|c|c|}
\hline \multicolumn{5}{|c|}{ SCL-90-R } & \multicolumn{5}{|l|}{ BSI-53 } & \multicolumn{5}{|c|}{ SCL-27 } \\
\hline \multirow[t]{2}{*}{ Scale } & \multicolumn{3}{|c|}{ Cronbach's alpha } & \multirow{2}{*}{$\begin{array}{l}\text { Number } \\
\text { of items }\end{array}$} & \multirow[t]{2}{*}{ Scale } & \multicolumn{3}{|c|}{ Cronbach's alpha } & \multirow{2}{*}{$\begin{array}{l}\text { Number } \\
\text { of items }\end{array}$} & \multirow[t]{2}{*}{ Scale } & \multicolumn{3}{|c|}{ Cronbach's alpha } & \multirow{2}{*}{$\begin{array}{l}\text { Number } \\
\text { of items }\end{array}$} \\
\hline & 1997 & 1999 & 2014 & & & 1997 & 1999 & 2014 & & & 1997 & 1999 & 2014 & \\
\hline SOMA & 0.90 & 0.89 & 0.93 & 12 & SOMA & 0.85 & 0.84 & 0.89 & 7 & VEG & 0.80 & 0.80 & 0.87 & 6 \\
\hline OCD & 0.81 & 0.83 & 0.90 & 10 & OCD & 0.76 & 0.79 & 0.86 & 6 & DYS & 0.74 & 0.78 & 0.81 & 4 \\
\hline INT & 0.80 & 0.82 & 0.87 & 9 & INT & 0.62 & 0.66 & 0.77 & 4 & SOP & 0.64 & 0.69 & 0.76 & 4 \\
\hline DEPR & 0.83 & 0.85 & 0.91 & 13 & DEPR & 0.73 & 0.75 & 0.82 & 6 & DEP & 0.59 & 0.63 & 0.66 & 4 \\
\hline ANX & 0.81 & 0.86 & 0.92 & 10 & ANX & 0.74 & 0.80 & 0.87 & 6 & & & & & \\
\hline HOST & 0.74 & 0.75 & 0.82 & 6 & HOST & 0.69 & 0.70 & 0.78 & 5 & & & & & \\
\hline $\mathrm{PHOB}$ & 0.74 & 0.78 & 0.87 & 7 & $\mathrm{PHOB}$ & 0.68 & 0.71 & 0.82 & 5 & AGO & 0.71 & 0.75 & 0.83 & 5 \\
\hline PARA & 0.74 & 0.75 & 0.84 & 6 & PARA & 0.72 & 0.72 & 0.81 & 5 & MIS & 0.64 & 0.69 & 0.76 & 4 \\
\hline PSYC & 0.79 & 0.78 & 0.90 & 10 & PSYC & 0.68 & 0.65 & 0.82 & 5 & & & & & \\
\hline GSI & 0.96 & 0.97 & 0.98 & 83 & GSI & 0.94 & 0.95 & 0.97 & 49 & GSI & 0.90 & 0.92 & 0.95 & 27 \\
\hline
\end{tabular}

SOMA Somatization, OCD Obsessive-Compulsive Disorder, INT Interpersonal Sensitivity, DEPR Depression, ANX Anxiety, HOST Hostility, PHOB Phobic Anxiety, PARA Paranoid Ideation, PSYC Psychoticism, GSI Global Severity Index, DEP Depressive Symptoms, DYS Dysthymic Symptoms, VEG Vegetative Symptoms, AGO Agoraphobic Symptoms, SOP Symptoms of Social Phobia, MIS Symptoms of Mistrust 
Table 2 Reliability of the SCL-90-R subscales and the shortened versions BSI, SCL-27, BSI-18, SCL-14 and SCL-K-9 in the Ukrainian general population (Continued)

\begin{tabular}{|c|c|c|c|c|c|c|c|c|c|c|c|c|c|c|}
\hline \multicolumn{5}{|l|}{ BSI-18 } & \multicolumn{5}{|c|}{ SCL-14 } & \multicolumn{5}{|c|}{ SCL-K-9 } \\
\hline \multirow[t]{2}{*}{ Scale } & \multicolumn{3}{|c|}{ Cronbach's alpha } & \multirow{2}{*}{$\begin{array}{l}\text { Number } \\
\text { of items }\end{array}$} & \multirow[t]{2}{*}{ Scale } & \multicolumn{3}{|c|}{ Cronbach's alpha } & \multirow{2}{*}{$\begin{array}{l}\text { Number } \\
\text { of items }\end{array}$} & \multirow[t]{2}{*}{ Scale } & \multicolumn{3}{|c|}{ Cronbach's alpha } & \multirow{2}{*}{$\begin{array}{l}\text { Number } \\
\text { of items }\end{array}$} \\
\hline & 1997 & 1999 & 2014 & & & 1997 & 1999 & 2014 & & & 1997 & 1999 & 2014 & \\
\hline SOMA & 0.84 & 0.84 & 0.89 & 6 & VEG & 0.85 & 0.84 & 0.88 & 4 & & & & & \\
\hline DEPR & 0.73 & 0.75 & 0.82 & 6 & DEP & 0.72 & 0.73 & 0.86 & 6 & & & & & \\
\hline \multirow[t]{2}{*}{ ANX } & 0.73 & 0.79 & 0.86 & 6 & & & & & & & & & & \\
\hline & & & & & AGO & 0.64 & 0.68 & 0.81 & 4 & & & & & \\
\hline GSI & 0.88 & 0.90 & 0.94 & 18 & GSI & 0.84 & 0.85 & 0.92 & 14 & GSI & 0.81 & 0.85 & 0.89 & 9 \\
\hline
\end{tabular}

and 1999 (in SCL-14). However, in the 2014 study, the only symptomatic dimension with an unsatisfactory level of reliability was a depressive symptoms scale in SCL-27.

It should be noted that the GSI in different versions of SCL-90-R ranged from 0.81 to 0.98 , indicating good reliability. In general, we can note satisfactory reliability for all versions of the symptomatic checklist.

\section{Factorial validity}

According to the RMSEA criteria $<0.06$ and CFI, TLI $>0.95$, all models have demonstrated satisfactory validity: the results of confirmatory factor analysis generally support the internal structure of symptomatic measures in the SCL-90-R (nine factors), BSI-53 (nine factors), SCL-27 (six factors), BSI-18 (three factors), SCL-14 (three factors) and SCL-90-R (a single factor) (Table 3). A comparison of models fit lower RMSEA and higher CFI and TLI in 2014 compared to the 1997 and 1999 studies. A three-factor model SCL14 and ten-factor model BSI-53 in the 2014 study are characterized by the lowest $\chi 2$ /DF ratios and the highest CFI and TLI values compared to other models; i.e. these versions of the symptomatic questionnaire demonstrate the best factorial validity.

In the 1997 study, the model fit of SCL-14 according to the $x 2$ DF ratio criterion was outside the acceptable limit $(\chi 2 / \mathrm{DF}=5,6)$. According to this criterion, poor model fit was also recorded in 1997 and 1999 with respect to SCL$90-\mathrm{R}(\chi 2 / \mathrm{DF}=6,5$ in 1997; $\chi 2$ / DF = 5,5 in 1999), and with respect to SCL-27 ( $\chi 2 / \mathrm{DF}=5,6$ in 1997). Satisfactory model fit based on all criteria $(\chi 2 / \mathrm{DF}<5$; RMSEA $<0.06$ and CFI, TLI $>0.95$ ) and during all three time periods characterizes BSI-53, BSI-18, and SCL-K-9.

Analysis of factor loadings on certain indicators of latent factors - symptomatic measures confirms satisfactory internal consistency (see Additional files 1, 2, 3, 4, 5 and 6 for details). The values of factor loadings in both the shortened and the full version of SCL-90-R exceeded 0.50, except for the indicator "nervousness" in the depression subscale in the SCL-90-R (1997 and

Table 3 Factor validity of SCL-90-R, BSI-53, SCL-27, BSI-18, SCL-14 and SCL-9-K in the Ukrainian general population: confirmatory factor analysis fit

\begin{tabular}{|c|c|c|c|c|c|c|c|c|c|c|c|c|c|c|c|c|c|c|}
\hline \multirow[t]{2}{*}{ Fit } & \multicolumn{3}{|c|}{ SCL-90-R } & \multicolumn{3}{|l|}{ BSI-53 } & \multicolumn{3}{|c|}{ SCL-27 } & \multicolumn{3}{|c|}{$\mathrm{BSI}-18$} & \multicolumn{3}{|c|}{ SCL-14 } & \multicolumn{3}{|c|}{ SCL-K-9 } \\
\hline & 1997 & 1999 & 2014 & 1997 & 1999 & 2014 & 1997 & 1999 & 2014 & 1997 & 1999 & 2014 & 1997 & 1999 & 2014 & 1997 & 1999 & 2014 \\
\hline X2 (DWLS) & 25299 & 21196 & 15672 & 6323 & 6117 & 4714 & 1844 & 1258 & 1243 & 575 & 530 & 543 & 415 & 279 & 183 & 111 & 118 & 121 \\
\hline DF & 3870 & 3870 & 3870 & 1280 & 1280 & 1280 & 309 & 309 & 309 & 132 & 132 & 132 & 74 & 74 & 74 & 27 & 27 & 27 \\
\hline$x_{2} / \mathrm{DF}$ & 6.5 & 5.5 & 4.0 & 4.9 & 4.8 & 3.7 & 6.0 & 4.1 & 4.0 & 4.4 & 4.0 & 4.1 & 5.6 & 3.8 & 2.5 & 4.1 & 4.4 & 4.5 \\
\hline$p$-value & 0.000 & 0.000 & 0.000 & 0.000 & 0.000 & 0.000 & 0.000 & 0.000 & 0.000 & 0.000 & 0.000 & 0.000 & 0.000 & 0.000 & 0.000 & 0.000 & 0.000 & 0.000 \\
\hline $\operatorname{RMSEA}(<0,06)$ & 0.057 & 0.052 & 0.042 & 0.048 & 0.047 & 0.038 & 0.053 & 0.042 & 0.040 & 0.044 & 0.041 & 0.040 & 0.051 & 0.040 & 0.027 & 0.042 & 0.044 & 0.042 \\
\hline CFI $(>0,95)$ & 0.967 & 0.979 & 0.995 & 0.980 & 0.984 & 0.996 & 0.977 & 0.989 & 0.996 & 0.991 & 0.993 & 0.997 & 0.988 & 0.993 & 0.999 & 0.993 & 0.995 & 0.997 \\
\hline TLI $(>0,95)$ & 0.966 & 0.978 & 0.995 & 0.979 & 0.983 & 0.996 & 0.974 & 0.987 & 0.996 & 0.990 & 0.992 & 0.997 & 0.985 & 0.991 & 0.998 & 0.991 & 0.993 & 0.997 \\
\hline
\end{tabular}


1999 studies). All factor loadings were statistically significant at the $1 \%$ level. There were strong correlations among all latent factors (see Additional files 7, 8, 9, 10 and 11 for details). For example, in the 2014 factor model of SCL-90-R the minimum and maximum correlation of certain symptomatic dimensions is 0.74 (somatization and hostility) and 0.99 (interpersonal sensitivity and paranoid tendencies); in BSI-53 - 0.78 (somatization and hostility, somatization and paranoid tendencies) and 0.99 (interpersonal sensitivity and phobic anxiety); in the SCL-27 - 0.78 (autonomic dysfunction and suspicion) and 0.98 (agoraphobia and social phobia); in BSI-18 - 0.86 (somatization and depression) and 0.98 (depression and anxiety); in the SCL-14 - 0.71 (autonomic disorder and agoraphobia) and 0.87 (agoraphobia and depression).

\section{Equivalence of the full and shortened versions of the SCL-90-R}

Correlations between the full and shortened versions of the SCL-90-R are expectedly very high. Spearman's Rho correlation coefficients vary in the range of 0.7-0.9 (Table 4). However, if we look at the difference in median values between similar components of SCL-90-R on the one hand, and BSI-53, SCL-27, BSI-18, SCL-14 and SCL-9-K on the other, it appears that in almost all cases, the difference in medians is statistically significant at the $5 \%$ level. Equivalence of medians between full and shortened versions of the questionnaire in all three analyzed studies is observed only for the somatization subscale in the BSI-18. It is noticeable that similar subscales in different versions differ not so much in a measure of central tendency, but in variance. Such differences cause statistically significant differences in small effect sizes. Analysis of the size effects (the so-called "scientific significance") shows that in all three studies the difference between symptomatic measures of SCL-90-R and BSI-53 is not significant (Vargha and Delaney's $\mathrm{A} \leq 0,56$ ). When comparing SCL-90-R and SCL-27, there is a small effect size for somatization and interpersonal sensitivity $(0.56<\mathrm{k} \leq 0,64$, in 1997, 1999 and 2014), whereas effect sizes on other symptomatic dimensions are insignificant. With regard to the comparison of SCL-90-R and such shortened versions as BSI-18, SCL-14, and SCL-9-R, in all three studies minor effect sizes were found, which indicates good equivalence.

When comparing full and shortened versions in the context of probable case prevalence according to Derogatis' criterion ( $T$-value for GSI $\geq 63$ ), in 1997 and 1999 only SCL-9-K shows prevalence estimates that go beyond the $95 \%$ confidence interval for the severe symptoms prevalence calculated by SCL-90-R (Fig. 1). In 2014, the variance of prevalence estimates in different versions of SCL-90-R is minimal.
According to the criterion of GSI > 1, the variance is greater: in 1997, 1999 and 2014 proportions of the population with severe symptoms based on BSI-18 and SCLK-9 were significantly higher at the $5 \%$ level than those based on SCL-90-R. In 1997, significantly higher proportions than in the full version of the SCL-90-R were also observed for SCL-14.

\section{Temporal stability}

If we consider reliability and validity of the five versions in terms of dynamics, there is acceptable consistency of scales and model fit of latent constructs in all three studies $(1997,1999,2014)$. Unfortunately, the secondary analysis does not allow us to estimate sensitivity to changes, as analyzed datasets of different years were not drawn from a single cohort.

\section{Discussion}

Overall, our analysis supported the reliability and the original factor structure of the Ukrainian version of SCL-90-R and its five shortened versions, as well as the acceptable equivalency of selected measures.

At the level of GSI analysis it is more profitable economically to use shortened versions, particularly SCL-K-9, which consists of nine points, and in whose favor are good reliability indicators, factor validity indicators and small effect sizes. For the analysis at the level of certain symptomatic dimensions, BSI-53 offers greater opportunities, where there are fewer questions than in the SCL-90-R, but there is the same 9-dimensional structure, satisfactory reliability and factorial validity. However, the 53-symptom questionnaire is quite cumbersome for annual monitoring surveys of the general population. According to the results of this paper, the best solution would be to use BSI-18, where the factorial structure of the three symptomatic measures is confirmed, satisfactory factor model fit is observed in all three studies (1997, 1999, and 2014), and there is a good internal consistency of the subscales.

In SCL-27, the problematic aspect for Ukraine is the lack of satisfactory reliability of the depression scale. However, lower reliability indicators in certain symptomatic measurements may result from sensitivity of the Cronbach's alpha criterion to the number of indicators within one symptomatic state $[6,37]$. The scale of depressive symptoms in the SCL-27 is calculated on the basis of a small number of indicators (four symptoms).

In the previous studies the lack of factor structure confirmation was a key target of criticism; in our opinion, such results could arise not so much because of the peculiarities of the country or of the analyzed groups, but because of the limitations in the sample size and the use of irrelevant methods, such as not accounting for ordinal scales of indicators in the SCL-90-R [23, 26-28]. Ukrainian study revealed that confirmatory factor 
Table 4 Medians (M) and interquartile ranges (IQR) of the SCL-90-R and the shortened versions: BSI-53, SCL-27, BSI-18, SCL-14, SCL-K-9 and the results of the Wilcoxon signed-rank test (W (p-value)), Vargha and Delaney's A effect sizes (ES) and Spearman's correlations (S.Rho) in the general population of Ukraine

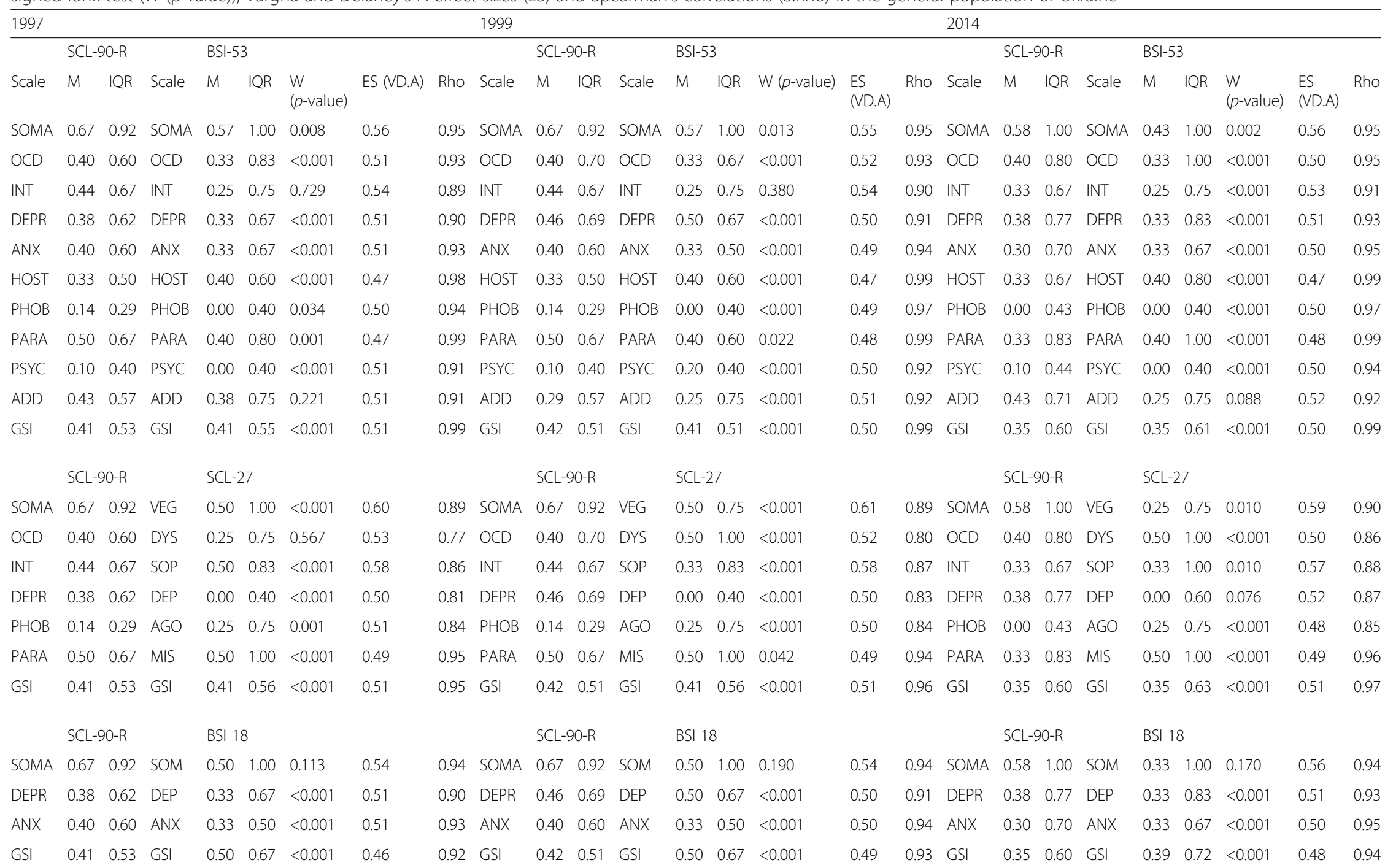


Table 4 Medians (M) and interquartile ranges (IQR) of the SCL-90-R and the shortened versions: BSI-53, SCL-27, BSI-18, SCL-14, SCL-K-9 and the results of the Wilcoxon signed-rank test (W ( $p$-value)), Vargha and Delaney's A effect sizes (ES) and Spearman's correlations (S.Rho) in the general population of Ukraine (Continued)

\begin{tabular}{|c|c|c|c|c|c|c|c|c|c|c|c|c|c|c|c|c|c|c|c|c|c|c|c|c|c|c|}
\hline \multirow[b]{2}{*}{ SOMA } & \multicolumn{3}{|c|}{ SCL-90-R } & \multicolumn{6}{|c|}{ SCL-14 } & \multicolumn{3}{|c|}{ SCL-90-R } & \multicolumn{6}{|c|}{ SCL-14 } & \multicolumn{3}{|c|}{ SCL-90-R } & \multicolumn{5}{|c|}{ SCL-14 } \\
\hline & 0.67 & 0.92 & SOM & 0.75 & 1.25 & $<0.001$ & 0.52 & 0.89 & SOMA & 0.67 & 0.92 & $\mathrm{SOM}$ & 0.75 & 1.00 & $<0.001$ & 0.51 & 0.90 & SOMA & 0.58 & 1.00 & SOM & 0.50 & 1.25 & $<0.001$ & 0.53 & 0.91 \\
\hline DEPR & 0.38 & 0.62 & DEP & 0.50 & 0.67 & $<0.001$ & 0.48 & 0.88 & DEPR & 0.46 & 0.69 & DEP & 0.50 & 0.83 & $<0.001$ & 0.47 & 0.90 & DEPR & 0.38 & 0.77 & DEP & 0.33 & 0.83 & $<0.001$ & 0.49 & 0.91 \\
\hline PHOB & 0.14 & 0.29 & $\mathrm{PHO}$ & 0.00 & 0.25 & $<0.001$ & 0.57 & 0.81 & $\mathrm{PHOB}$ & 0.14 & 0.29 & $\mathrm{PHO}$ & 0.00 & 0.25 & $<0.001$ & 0.57 & 0.83 & $\mathrm{PHOB}$ & 0.00 & 0.43 & $\mathrm{PHO}$ & 0.00 & 0.25 & $<0.001$ & 0.54 & 0.88 \\
\hline \multirow[t]{2}{*}{ GSI } & 0.41 & 0.53 & GSI & 0.43 & 0.64 & $<0.001$ & 0.49 & 0.90 & GSI & 0.42 & 0.51 & GSI & 0.50 & 0.64 & $<0.001$ & 0.47 & 0.90 & GSI & 0.35 & 0.60 & GSI & 0.36 & 0.64 & $<0.001$ & 0.51 & 0.93 \\
\hline & \multicolumn{3}{|c|}{ SCL-90-R } & \multicolumn{6}{|c|}{ SCL-K-9 } & \multicolumn{3}{|c|}{ SCL-90-R } & \multicolumn{6}{|c|}{ SCL-K-9 } & \multicolumn{3}{|c|}{ SCL-90-R } & \multicolumn{5}{|c|}{ SCL-K-9 } \\
\hline GSI & 0.41 & 0.53 & GSI & 0.56 & 0.89 & $<0.001$ & 0.43 & 0.91 & GSI & 0.42 & 0.51 & GSI & 0.56 & 0.78 & $<0.001$ & 0.41 & 0.91 & GSI & 0.35 & 0.60 & GSI & 0.50 & 0.78 & $<0.001$ & 0.45 & 0.91 \\
\hline
\end{tabular}

SOMA Somatization, OCD Obsessive-Compulsive Disorder, INT Interpersonal Sensitivity, DEPR Depression, ANX Anxiety, HOST Hostility, PHOB Phobic Anxiety, PARA Paranoid Ideation, PSYC Psychoticism, GSI Global Severity Index, DEP Depressive Symptoms, DYS Dysthymic Symptoms, VEG Vegetative Symptoms, AGO Agoraphobic Symptoms, SOP Symptoms of Social Phobia, MIS Symptoms of Mistrust 


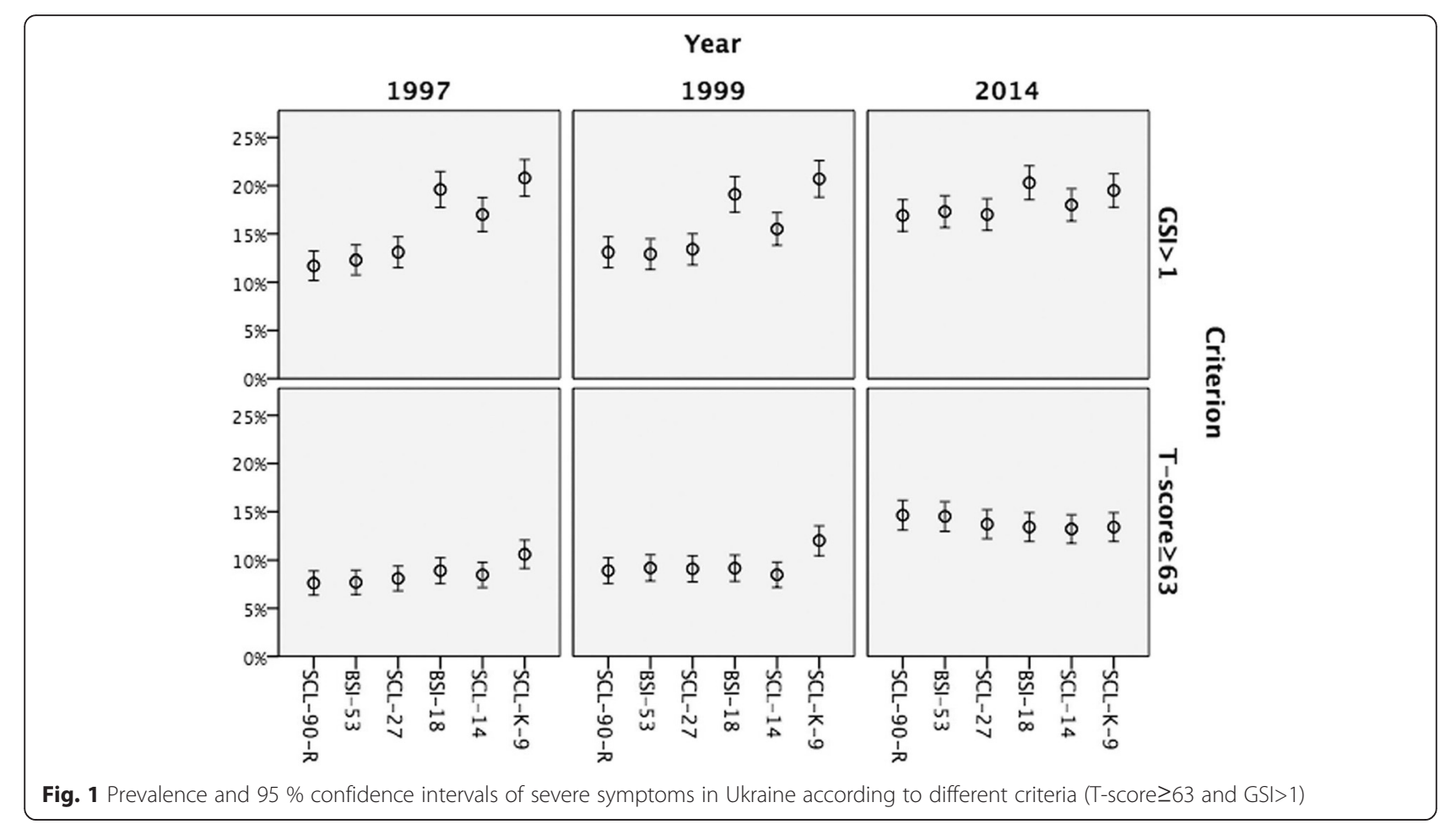

analysis on 2014 data has confirmed the structure of symptomatic scales in both full and shortened versions of the SCL-90-R. However, in the 1997 and 1999 studies, which had a smaller sample size than the 2014 study, a number of models (SCL-90-R, SCL-27, and SCL-14) have shown unsatisfactory fit according to the $\mathrm{X}$-square criterion, although on other fit indices (RMSEA, CFI, TLI) satisfactory results have been obtained.

Analysis of the equivalence of shortened and full versions of the SCL-90-R has shown the presence of small effect sizes, which is consistent with the results of validation in other countries [11, 22]. On the other hand, analysis of probable cases prevalence by different criteria ( $T$-score $>63$ or GSI $>1$ ) indicates that SCL-K-9 shows higher prevalence of severe symptoms. However, the analysis of equivalence of the shortened versions and SCL-90-R for assessing the prevalence of distress requires further investigation, in particular clarification of the criteria for threshold values of probable cases in Ukraine as well as in certain population groups.

Despite a generally positive assessment of the validation of SCL-90-R as well as its shortened versions in Ukraine, a number of limitations should be noted. Firstly, SCL-90-R wasn't the main objective of the study in any of the three analyzed surveys. The symptomatic checklist was located at the end of the monitoring questionnaire, which included a wide range of issues. This could affect the completion of the questionnaire due to fatigue of the respondents. Secondly, the 1997/1999 and 2014 studies, although they are similar in design (all three are cross-sectional and representative of the population, and used the same method of data collection), they have differences in a number of procedural aspects: sample building, a set of cluster profiles, period of field stage, and being carried out by different organizations for different objectives. Thirdly, all the periods when SCL-90- $\mathrm{R}$ was used in Ukraine among the general population, were characterized as periods of severe social crisis. 1997 and 1999 were characterized by significant economic difficulties, and 2014 - by the political crisis and military conflict in the east of the country, which involved a number of challenges for the whole country. There is a lack of a comparable assessment conducted during a relatively prosperous period (for example, in the 2000s before the financial crisis) that would allow for evaluation of the sensitivity of the questionnaire to such changes. Fourthly, convergent and discriminant validity of the full and shortened versions in Ukraine remain questionable as none of the appropriate alternative screening tools were used simulteniously with the SCL-90-R.

Among the strengths of our study is the fact that the results can be generalized to the entire population of Ukraine. The presence of three waves of the study at different times allowed us to check the temporal stability of the factor structure and reliability of the tool during different stages of social and economic development of the country. Another strong point of the study is the use of CFA with polychoric correlations, which allowed for improvement of the model fit. A certain advantage of the analysis is the use of non-parametric methods of 
analysis, in particular the description of data through the median and interquartile distance, using Spearman's Rho correlations and Vargha and Delaney's A effect sizes. The traditional approach to the analysis of certain symptomatic dimensions usually includes the calculation of averages, standard deviations, Pearson's correlations and parametric effect sizes. Our study has shown that all symptomatic measurements have distributions which deviate from normal even in large samples, so the use of non-parametric methods for the validation SCL-90-R is more appropriate.

Prospects for further validation of SCL-90-R studies in Ukraine suggest evaluation of the discriminant and convergent validity using alternative questionnaires measuring psychological distress, conducting cohort studies to determine the sensitivity of the questionnaire to social changes and studying the relevant thresholds for determining probable cases of psychological distress as well as its symptomatic dimensions.

\section{Conclusion}

This validation study of the full and shortened versions of the SCL-90-R has shown that SCL-K-9 might be an optimal solution for assessing general distress in national population monitoring studies in Ukraine. If it is necessary to analyze certain symptomatic dimensions of distress, using BSI-18 is recommended.

\section{Additional files}

Additional file 1: Confirmatory factor analysis of the SCL-90-R in the Ukrainian general population: factor loadings. Results of confirmatory factor analysis. Factor loadings for nine dimensions of the SCL-90-R in 1997, 1999 and 2014 studies that are representative of the Ukrainian population. (XLSX $12 \mathrm{~kb})$

Additional file 2: Confirmatory factor analysis of the BSI-53 in the Ukrainian general population: factor loadings. Results of confirmatory factor analysis. Factor loadings for nine dimensions of the BSI-53 in 1997, 1999 and 2014 studies that are representative of the Ukrainian population. (XLSX $11 \mathrm{~kb}$ )

Additional file 3: Confirmatory factor analysis of the SCL-27 in the Ukrainian general population: factor loadings. Results of confirmatory factor analysis. Factor loadings for six dimensions of the SCL-27 in 1997, 1999 and 2014 studies that are representative of the Ukrainian population. (XLSX $10 \mathrm{~kb}$ )

Additional file 4: Confirmatory factor analysis of the BSI-18 in the Ukrainian general population: factor loadings. Results of confirmatory factor analysis. Factor loadings for three dimensions of the BSI-18 in 1997, 1999 and 2014 studies that are representative of the Ukrainian population. (XLSX $9 \mathrm{~kb})$

Additional file 5: Confirmatory factor analysis of the SCL-14 in the Ukrainian general population: factor loadings. Results of confirmatory factor analysis. Factor loadings for three dimensions of the SCL-14 in 1997, 1999 and 2014 studies that are representative of the Ukrainian population. (XLSX $9 \mathrm{~kb})$

Additional file 6: Confirmatory factor analysis of the SCL-K-9 in the Ukrainian general population: factor loadings. Results of confirmatory factor analysis. Factor loadings for the SCL-K-9 in 1997, 1999 and 2014 studies that are representative of the Ukrainian population. (XLSX $8 \mathrm{~kb}$ )
Additional file 7: Confirmatory factor analysis of the SCL-90-R in the Ukrainian general population: correlations between latent factors. Results of confirmatory factor analysis. Correlations between the latent variables measured by SCL-90-R items in 1997, 1999 and 2014 studies that are representative of the Ukrainian population. (XLSX $10 \mathrm{~kb}$ )

Additional file 8: Confirmatory factor analysis of the BSI-53 in the Ukrainian general population: correlations between latent factors. Results of confirmatory factor analysis. Correlations between the latent variables measured by BSI-53 items in 1997, 1999 and 2014 studies that are representative of the Ukrainian population. (XLSX $11 \mathrm{~kb}$ )

Additional file 9: Confirmatory factor analysis of the SCL-27 in the Ukrainian general population: correlations between latent factors. Results of confirmatory factor analysis. Correlations between the latent variables measured by SCL-27 items in 1997, 1999 and 2014 studies that are representative of the Ukrainian population. (XLSX $10 \mathrm{~kb}$ )

Additional file 10: Confirmatory factor analysis of the BSI-18 in the Ukrainian general population: correlations between latent factors. Results of confirmatory factor analysis. Correlations between the latent variables measured by BSI-18 items in 1997, 1999 and 2014 studies that are representative of the Ukrainian population. (XLSX $9 \mathrm{~kb}$ )

Additional file 11: Confirmatory factor analysis of the SCL-14 in the Ukrainian general population: correlations between latent factors. Results of confirmatory factor analysis. Correlations between the latent variables measured by SCL-14 items in 1997, 1999 and 2014 studies that are representative of the Ukrainian population. (XLSX $9 \mathrm{~kb}$ )

\section{Acknowledgements}

We would like to thank Prof. Dr. Evgeniy Golovakha, Vice Director of Science of the Institute of Sociology, National Academy of Sciences in Ukraine, for his patient guidance, encouragement and advice. Completing this study would have been all the more difficult were it not for the support provided by Olga Balakireva, the Head of the Department for Monitoring of Social and Economic Transformations, Institute for Economics and Forecasting, National Academy of Sciences in Ukraine. We must express our gratitude to Dr. Evelyn J. Bromet, Distinguished Professor of the Department of Psychiatry and Behavioral Science, State University of New York at Stony Brook, who introduced Symptom Checklist-90 Revised within the framework of the Stony Brook-Kyiv Chernobyl Project, a collaboration between US investigators and independent scientists in Ukraine. This project contributed to the application of SCL-90-R in Ukrainian national surveys. Finally, we would like to thank Dr. Nataliia Panina (deceased) for her work on developing the Ukrainian version of SCL-90-R.

\section{Funding}

We gratefully acknowledge support from the National Institutes of Health [grant number D43 TW000233] funded by the Fogarty International Center and the National Academy of Sciences in Ukraine within the research project "Development and adaptation of data measurement tools for public sociological surveys in the transforming society" [grant number 0114U003415].

\section{Availability of data and materials}

Datasets supporting our results can be found in the Institute of Sociology, National Academy of Sciences in Ukraine (1997 and 1999 studies) and in the Department for Monitoring of Social and Economic Transformations, Institute for Economy and Forecasting, National Academy of Sciences in Ukraine (2014 study). The datasets are in Ukrainian. According to the policy of both institutes, data is not deposited in publicly available repositories. However, it might be available under request.

\section{Authors' contributions}

YS carried out the validation methodology, performed the statistical analysis, and helped to interpret the data and to draft the manuscript. SD participated in the design of the study and the literature review, analyzed the data and drafted the first version of the manuscript. Both authors read and approved the final manuscript.

\section{Competing interests}

The authors declare that they have no competing interests. 


\section{Consent for publication}

Not applicable.

\section{Ethics approval and consent to participate}

Independent ethics committee at the Sociological Association of Ukraine ruled that no formal ethics approval was required in this particular case because the study represents a secondary analysis of data that was completely anonymous when provided to the authors, and it was impossible to identify participants from any resulting reports. This complies with the Code of Professional Ethics of Sociologist adopted by the 5th Congress of the Sociological Association of Ukraine [38]. Informed consent to participate in the study was obtained from participants.

\section{Author details}

${ }^{1}$ Senior Scientific Associate, Department for Monitoring of Social and Economic Transformations, Institute for Economics and Forecasting, National Academy of Sciences, Kiev, Ukraine. ${ }^{2}$ Senior Scientific Associate, Department of Methodology and Methods of Sociology, Institute of Sociology, National Academy of Sciences, Kiev, Ukraine.

\section{Received: 21 January 2016 Accepted: 18 August 2016}

Published online: 26 August 2016

\section{References}

1. Derogatis LR. SCL-90-R: Administration, Scoring of Procedures Manual-Il for the R (evised) Version and Other Instruments of the Psychopathology Rating Scale Series: Clinical Psychometric Research Incorporated. 1992.

2. Bromet EJ, Goldgaber D, Carlson G, Panina N, Golovakha E, Gluzman SF, Gilbert T, Gluzman D, Lyubsky S, Schwartz JE. Children's well-being 11 years after the Chornobyl catastrophe. Arch Gen Psychiatry. 2000;57(6):563-71.

3. Derogatis LR, Spencer P. Brief symptom inventory: BSI. Upper Saddle River: Pearson; 1993.

4. Hardt J, Gerbershagen HU. Cross-validation of the SCL-27: A short psychometric screening instrument for chronic pain patients. Eur J Pain. 2001;5(2):187-97.

5. Derogatis LR. Brief symptom inventory 18. Minneapolis: NCS Pearson; 2000

6. Prinz U, Nutzinger DO, Schulz H, Petermann F, Braukhaus C, Andreas S. Comparative psychometric analyses of the SCL-90-R and its short versions in patients with affective disorders. BMC Psychiatry. 2013;13:104.

7. Harfst T, Koch U. Kurtz von Aschoff C, Nutzinger D, Rüddel H, Schulz H: Entwicklung und Validierung einer Kurzform der Symptom Checklist-90-R. DRV-Schriften. 2002;33:71-3.

8. Klaghofer R, Brähler E. Konstruktion und Teststatistische Prüfung einer Kurzform der SCL-90-R, Zeitschrift für Klinische Psychologie, Psychiatrie und Psychotherapie. 2001.

9. Martinez-Azumendi O, Fernandez-Gomez C, Beitia-Fernandez M. [Factorial variance of the SCL-90-R in a Spanish out-patient psychiatric sample]. Actas Esp Psiquiatr. 2001;29(2):95-102.

10. Grande TL, Newmeyer MD, Underwood LA, Williams CR. Path Analysis of the SCL-90-R: Exploring Use in Outpatient Assessment. Measurement and Evaluation in Counseling and Development. 2014;47(4):271-90.

11. Paap MC, Meijer RR, Cohen-Kettenis PT, Richter-Appelt H, de Cuypere G, Kreukels BP, Pedersen G, Karterud S, Malt UF, Haraldsen IR. Why the factorial structure of the SCL-90-R is unstable: comparing patient groups with different levels of psychological distress using Mokken Scale Analysis. Psychiatry Res. 2012;200(2-3):819-26.

12. Paap M, Meijer RR, Van Bebber J, Pedersen G, Karterud S, Hellem FM, Haraldsen IR. A study of the dimensionality and measurement precision of the SCL-90-R using item response theory. Int J Methods Psychiatr Res. 2011;20(3):e39-55.

13. Rauter UK, Leonard CE, Swett CP. SCL-90-R factor structure in an acute, involuntary, adult psychiatric inpatient sample. J Clin Psychol. 1996;52(6):625-9.

14. Bonynge ER. Unidimensionality of SCL-90-R scales in adult and adolescent crisis samples. J Clin Psychol. 1993;49(2):212-5.

15. Zack M, Toneatto T, Streiner DL. The SCL-90 factor structure in comorbid substance abusers. J Subst Abuse. 1998;10(1):85-101.

16. Bech P, Bille J, Møller S, Hellström L, Østergaard SD. Psychometric validation of the Hopkins Symptom Checklist (SCL-90) subscales for depression, anxiety, and interpersonal sensitivity. J Affect Disord. 2014;160:98-103.

17. Clark A, Friedman MJ. Factor structure and discriminant validity of the SCL-90 in a veteran psychiatric population. J Pers Assess. 1983;47(4):396-404.
18. Ransom D, Ashton K, Windover A, Heinberg L. Internal consistency and validity assessment of SCL-90-R for bariatric surgery candidates. Surg Obes Relat Dis. 2010;6(6):622-7.

19. Derogatis LR, Rickels K, Rock AF. The SCL-90 and the MMPI: a step in the validation of a new self-report scale. Br J Psychiatry. 1976;128:280-9.

20. Fortin MF, Coutu-Wakulczyk G, Engelsmann F. Contribution to the validation of the SCL-90-R in French-speaking women. Health Care Women Int. 1989;10(1):27-41.

21. Olsen LR, Mortensen EL, Bech P. The SCL-90 and SCL-90R versions validated by item response models in a Danish community sample. Acta Psychiatr Scand. 2004;110(3):225-9.

22. Holi MM, Sammallahti PR, Aalberg VA. A Finnish validation study of the SCL-90. Acta Psychiatr Scand. 1998;97(1):42-6.

23. Schmitz N, Hartkamp N, Kiuse J, Franke GH, Reister G, Tress W. The Symptom Check-List-90-R (SCL-90-R): a German validation study. Qual Life Res. 2000;9(2):185-93.

24. Urban R, Kun B, Farkas J, Paksi B, Kokonyei G, Unoka Z, Felvinczi K, Olah A, Demetrovics Z. Bifactor structural model of symptom checklists: SCL-90-R and Brief Symptom Inventory (BSI) in a non-clinical community sample. Psychiatry Res. 2014;216(1):146-54.

25. Tomioka M, Shimura M, Hidaka M, Kubo C. The reliability and validity of a Japanese version of symptom checklist 90 revised. Bio Psycho Social Med. 2008;2(1):1-8.

26. Prunas A, Sarno I, Preti E, Madeddu F, Perugini M. Psychometric properties of the Italian version of the SCL-90-R: a study on a large community sample. Eur Psychiatry. 2012:27(8):591-7.

27. Vassend $\mathrm{O}$, Skrondal $\mathrm{A}$. The problem of structural indeterminacy in multidimensional symptom report instruments. The case of SCL-90-R. Behav Res Ther. 1999;37(7):685-701.

28. Wongpakaran T, Wongpakaran N, Boripuntakul T. Symptom checklist-90 (SCL-90) in a Thai sample. J Med Assoc Thai. 2011;94(9):1141-9.

29. Chapman LK, Petrie J, Vines L. Factor structure analysis of the SCL-90-R in a community-based sample of African American women. Psychiatry Res. 2012;199(2):140-4.

30. Müller JM, Postert C, Beyer T, Furniss T, Achtergarde S. Comparison of eleven short versions of the Symptom Checklist 90-Revised (SCL-90-R) for use in the assessment of general psychopathology. J Psychopathol Behav Assess. 2009;32(2):246-54.

31. Peterson RA. A meta-analysis of Cronbach's coefficient alpha. J Consum Res. 1994;381-391.

32. Yu C-Y. Evaluating cutoff criteria of model fit indices for latent variable models with binary and continuous outcomes. University of California Los Angeles; 2002

33. Vargha A, Delaney HD. A critique and improvement of the $\mathrm{CL}$ common language effect size statistics of McGraw and Wong. J Educ Behav Stat. 2000;25(2):101-32

34. Cohen J. Statistical power analysis for the behavioral sciences. Routledge academic. Mahwah, NJ: Lawrence Erlbaum Associates; 2013.

35. Derogatis LR, Fitzpatrick M. The SCL-90-R, the Brief Symptom Inventory (BSI), and the BSI-18. 2004.

36. Olsen LR, Mortensen EL, Bech P. Mental distress in the Danish general population. Acta Psychiatr Scand. 2006;113(6):477-84.

37. Eisen SV, Dill DL, Grob MC. Reliability and validity of a brief patient-report instrument for psychiatric outcome evaluation. Psychiatr Serv. 1994;45(3):242-7.

38. Panina N. Professional Ethics and Sociology in Ukraine (On Adoption of the Code of Professional Ethics of Sociologist by the Sociological Association of Ukraine), Ukrainian Sociological Review 2004-2005. 2007. 